\title{
Quantum dots in nanomedicine: recent trends, advances and unresolved issues
}

\author{
Yuri Volkov \\ Department of Clinical Medicine, School of Medicine and AMBER Centre, Trinity College, Dublin 8, Ireland
}

\section{A R T I C L E I N F O}

\section{Article history:}

Received 29 June 2015

Accepted 7 July 2015

Available online $\mathrm{xxx}$

\section{Keywords:}

Quantum dots

Nanomedicine

Nanoparticles

Medical imaging

Diagnostics

Theranostics

\begin{abstract}
A B S T R A C T
The review addresses the current state of progress in the use of ultra-small nanoparticles from the category of quantum dots (QDs), which presently embraces a widening range of nanomaterials of different nature, including "classical" semiconductor groups III-V and II-VI nanocrystals, along with more recently emerged carbon, silicon, gold and other types of nanoparticles falling into this class of nanomaterials due to their similar physical characteristics such as small size and associated quantum confinement effects. A diverse range of QDs applications in nanomedicine has been extensively summarised previously in numerous publications. Therefore, this review is not intended to provide an all-embracing survey of the well documented QDs uses, but is rather focused on the most recent emerging developments, concepts and outstanding unresolved problematic and sometimes controversial issues. Over 125 publications are overviewed and discussed here in the context of major nanomedicine domains, i.e. medical imaging, diagnostics, therapeutic applications and combination of them in multifunctional theranostic systems.
\end{abstract}

(c) 2015 Elsevier Inc. All rights reserved.

\section{Quantum dots in nanomedicine: recent trends}

Within a steadily increasing database of diverse nanomaterials reported as suitable for applications in nanomedicine [1-10], quantum dots (QDs) deservedly occupy a special niche as nanoparticles with a unique track record full of high expectations, dramatic pitfalls and often controversial experimental evidence. From the early optimistic aspirations of them as breakthrough tools for multipurpose in vitro and in vivo applications [11-17], they have been subject to a period of partial relinquishment following the sombre realisation that in the original unmodified state QDs did not stand a chance to deserve a unanimous approval as imaging or drug delivery vehicles in humans, due to their intrinsic toxicity and lack of biodegradability perspective. The disappointingly low yield of exploitable outputs following the initial rounds of heavy investments in the field has also contributed to the overall decline in research productivity metrics. The analysis of the recent ten years' trends in publications number in this area related to the QDs medical applications in vivo in general (Fig. $1 \mathrm{~A}$ ), as well as for imaging and diagnostics purposes (Fig. $1 \mathrm{~B}, \mathrm{C}$ ) according to Thomson Reuters Web of Science ${ }^{\mathrm{TM}}$ database clearly reflects such "cooling down" period after 2010 resulting in a significant reduction in the initially steady exponential growth of publication rates. This

E-mail address: yvolkov@tcd.ie. trend has been partially or completely reversed by 2014 and it is intriguing whether we will see it sustained in 2015 and beyond.

Such returning enthusiasm has been reinvigorated by a number of objective tendencies in the related technological developments, including the arrival of innovative nanotechnology-enabled tools for diagnostic and ex vivo imaging applications [18,19], the arrival of new types of QDs of alternative nature offering the opportunities of reducing the undesirable QDs-associated toxicity and side effects, such as carbon, graphene $[20,21]$, silicon quantum dots and nanodiamonds [22], hence refocusing the attention of scientists pursuing the progress in this field in academia, research institutions and industry alike. Selective optimisation and surface chemistry manipulation of semiconductor QDs hold the potential for their multimodal applications such as ultrasensitive detection in diagnostic systems, as well as drug delivery approaches, combining precision targeting, drug delivery and imaging in a single assay [18]. Advances in single molecule imaging using multi-color and threedimensional QD tracking, have enabled the in vivo observation of biological processes at molecular level resolution, including whole animal studies [19].

\section{Advances in the "anatomy" of biocompatible quantum dots}

Since the time when the term "quantum dot" has been coined by Reed et al. [23], quantum dots (QDs) have been commonly 

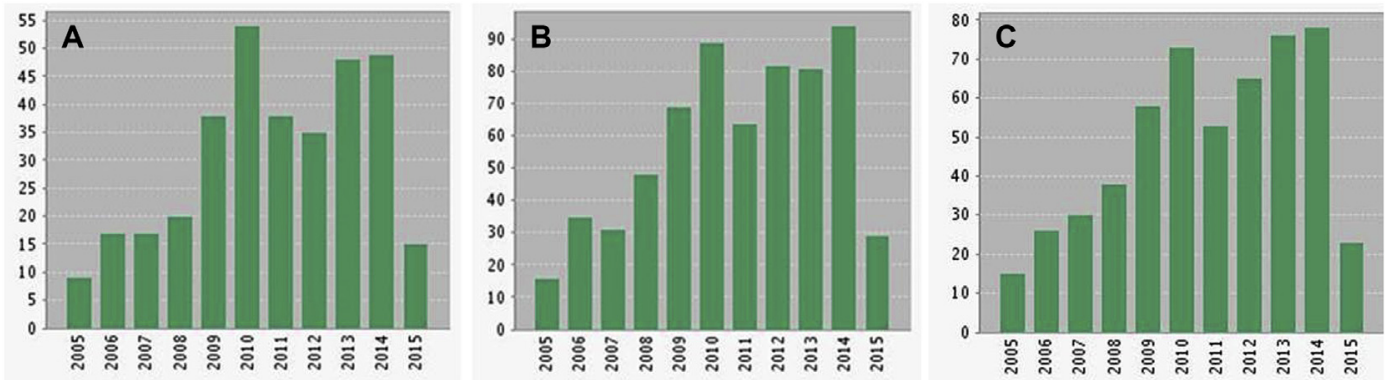

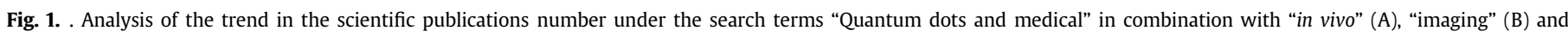

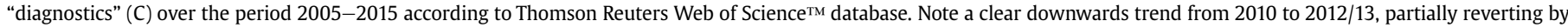
2014.

perceived as inorganic semiconductor nanocrystals typically synthetized from groups III-V and II-VI elements of the Periodic Table. However, recent developments in the nanotechnology area and biomedical applications of nanomaterials make us explicitly note that despite remaining unchanged in most widely accessible public information domains, the contemporary definition of QDs must be substantially broadened to include wider categories of nanoparticles more recently introduced by the researchers and based on carbon, silicon, gold, molybdenum sulphate and similar materials, all of which display the quantum confinement phenomenon associated with a dramatic change of electrons' behaviour kept at the boundaries of the Bohr radius in ultra-small objects with size below $10 \mathrm{~nm}$. Due to their extremely small size (compared to most cellular, subcellular structures and many biomolecules), they can exert their interactions with biological structures at the near atomic scale [24]. Nonetheless, the comprehensive analysis of the available scientific reports in the field shows that the more "classical" semiconductor III-V and II-VI nanocrystals still occupy the widest niche in biological research and medical applications for several objective reasons. Firstly, the sheer amount of reliable and reproducible data related to these QDs type accumulated over decades significantly exceeds such on the other QDs types. Secondly, they possess truly outstanding physico-chemical properties clearly distinguishing them from other nanoparticles. Some of the inorganic semiconductor QDs have already been successfully utilised in biomedical applications. In addition, the expanding use of such QDs in technical applications, devices and products dictates the necessity of a continuous thorough assessment of the associated risks and hazards for people, should they be released in the environment and be subject to uncontrolled human population exposure.

In general, to exert the features ideally suitable for medical uses, all types of QDs should be as small as possible for deeper organ, tissue and intracellular barriers penetration and on the other hand - efficient excretion if introduced in vivo, be as bright and stable as possible for more sensitive and reliable detection and last but not the least, show minimal signs of toxicity if applied in vivo or introduced into human organism following an unintentional exposure.

"Classical" semiconductor QDs, in addition to their dimensional limits (common core diameters in the range 1-10 nm), display unique optical characteristics due to the above mentioned quantum confinement effect [25]. The optical properties which deservedly positioned QDs as an outstanding class of fluorescent probes for biomedical imaging include, among the others, broad absorption spectra enabling simultaneous excitation of multiple fluorescence colours [26], very narrow and size-tuneable light emission bands $[27,28]$ along with high resistance to photo-bleaching compared to the conventional organic fluorophores [29].
However, in order to be useful for biomedical purposes, QDs commonly undergo a multi-step synthetic and preparatory process ensuring solubility and biocompatibility [24] which we briefly describe below. The first step in the process of QD core synthesis involves injection of liquid precursors into solvents at high temperature, with a subsequent deposition of an inorganic shell protecting the optical properties of the core. This increases the quantum yield to nearly $80 \%[29,30]$. The emission efficiency in QDs-based systems can be further enhanced using optimally designed plasmonic gold-derived structures [31]. However, the ex synthesis QDs are non-polar and therefore insoluble in water. They must be rendered water soluble in order to be utilised in biological systems [32]. Hence the surface of the nanoparticle is further covered with solubilisation ligands, making them transferrable to the aqueous environment. Various solubilisation ligands such as thiol-containing molecules, peptides and polymerised silica shells with polar groups are used [33]. The resulting charge of QDs in relation to the surrounding medium referred to as the zeta potential (i.e. the electro-kinetic potential in colloidal systems) has a pronounced effect on the QDs interactions with the biological structures. Alternatively, QDs can be made biocompatible by capping the hydrophobic shell with hydrophilic ligands such as dihydrolipoic acid [34]. In addition to ligand exchange, QDs with hydrophobic surface ligands can be solubilized by encapsulation in amphiphilic polymers [35,36] and phospholipid micelles [37]. Gaponik et al. offered a valuable alternative method of direct aqueous synthesis of QDs [38] which received a wide recognition for its safety, efficiency and reliability.

Among the recently emerged types of nanoparticles for biomedical use, graphene QDs are a promising class of nanomaterials with size dependent optical properties similar to semiconductor QDs, e.g. tuneable emission bands and amenability to targeted bio-functionalization. Doping of these graphene QDs with heteroatoms can further attenuate their optical qualities, permitting to achieve the photoluminescence quantum yield of up to $70 \%$ [39]. Chua et al. have successfully used fullerene C60 to produce very small graphene QDs of $2-3 \mathrm{~nm}$ which demonstrated good dispersion qualities in aqueous suspension and exhibited strong luminescence emission at $460 \mathrm{~nm}$, which could be shifted to red or blue areas of spectrum following customised chemical treatment with hydrazine hydrate and hydroxylamine [40]. Very recently, Dai et al. reported the synthesis and potential application of have molybdenum disulphide QDs of less than $10 \mathrm{~nm}$ size with excitation-independent blue photoemission, quantum yield approaching $10 \%$ and fluorescence stability over a $\mathrm{pH}$ range between from 4 to 10 , making them good candidates for multiphoton biomedical imaging [41]. The range of QDs with nanomedical application potential has been expanded with the introduction of silicon nanoparticles with tuneable emission properties over 
$470-560 \mathrm{~nm}$ wavelength range and $25 \%$ quantum yield. These Siderived QDs have shown good shelf life and photostability, retaining a strong and stable fluorescent signal over $3 \mathrm{~h}$ under a high power UV illumination, in contrast to the deteriorating fluorescence quenching of conventional organic dyes and group II-VI QDs over $2 \mathrm{~h}$ under the identical experimental conditions [42]. Gold QDs exhibit distinctive optical and magnetic properties compared to more commonly used gold nanoparticles. However, their unfavourable interactions with live cells and insufficient stability in aqueous media prevented their wider application in biomedical studies. Hembury et al. provided an interesting synthetic pathway integrating gold QDs within a mesoporous silica shell, alongside larger gold nanoparticles within the shell's central cavity. Such "quantum rattles", as the authors named them, were stable in aqueous solutions, didn't show signs of cytotoxicity, preserved the attractive near-infrared photonics and paramagnetic features of gold QDs [43]. Zheng et al. have earlier introduced fluorescent, water-soluble and size-tuneable over the visible and near-infrared spectra gold QDs, thereby offering the alternative light-emitting probes for biomedical imaging [44].

The range of the nanoparticles falling under the category of QDs for biomedical utilization is currently on a steady increase.

\section{Quantum dots for in vitro biomedical experimental systems}

Prior to discussing the applications of QDs as imaging and diagnostic tools in nanomedicine, it must be stressed that albeit imaging conditions in experimental settings in vitro and clinical diagnostic imaging are usually very different and not all the innovative nanoprobes eventually find their way into realistic nanomedical uses, we felt it essential to overview here the current developments in cellular and tissue models, as these might bear a potential for pre-clinical and clinical translation, even if in a distant future.

The design of QDs for successful imaging of specific tissue, cellular and subcellular targets is achieved via their functionalization with biologically active molecules, such as antibodies, drugs, peptides and small molecular moieties, micelles, etc. $[45,46]$. Biofunctionalization allows for improved stability and biocompatibility of QDs, enabling them to be used for specific in vitro biological labelling. Avidin [47], serotonin [48], and chitosan [49] are among many of those used to date to target specific cell receptors and proteins. Peptide coated CdSe/ZnS QDs were successfully applied to specifically target chimeric proteins expressed on the membranes of live HeLa cells [50]. QDs have also been used as probes to monitor individual synaptic receptors in living neural circuits [51]. Goa et al. showed that optically encoded beads using mesoporous polystyrene beads and surfactant-coated semiconductor QDs could be suitable for detection with a standard flow cytometer at 1000 beads/s [52]. Pathak et al. demonstrated the use of hydroxylated QDs as luminescent probes for fluorescent in situ hybridization [53]. Bio-functionalized QDs can be utilized for detection of genomic aberrations of cancer genes by this technique [54]. Zhu et al. developed semiconductor quantum dot-conjugated antibodies to label Cryptosporidium parvum and Giardia lamblia [55]. Bacterial strains that are slow growing (e.g., Mycobacteria), or highly infectious (e.g., Bacillus anthracis) could be detected using phage conjugated streptavidin-coated QDs [56]. Han et al. achieved multicolor optical coding for biological assays by embedding different-sized CdSe/ZnS QD into polymeric microbeads at precisely controlled ratios and utilized it in DNA hybridization studies [57]. Antibody conjugated CdSe/ZnS core-shell QD were utilized to perform multiplexed fluorescent immunoassays for the simultaneous detection of cholera toxin, ricin, Shiga-like toxin 1, and staphylococcal enterotoxin B [14]. The unique optoelectronic properties of QDs give them major advantages over the existing organic dyes not only as fluorescent labels, but also as lightemitting sensing probes. ZnS-capped CdSe QDs have been shown to be sensitive to environmental factors such as $\mathrm{pH}$ and the presence of divalent cations [58]. QDs-based sensors utilizing the fluorescent resonance energy transfer (FRET) technique deserve a special interest, as they can be applied as ratiometric cellular probes for the measurement of $\mathrm{pH}$, oxygen, metal ions and enzymatic activity $[13,59]$, including caspases as important indicators of cell health and apoptotic processes [60].

QDs also offer an opportunity to investigate the fine organisation of human cells at the cytoplasmic and subcellular organelle levels. Williams et al. studied the compartmentalization of sizetuned luminescent semiconductor nanocrystal QDs in four distinctive cell lines and found that each cell line displays its own cut off size thresholds reflecting cell-type-determined cytoplasmic compartmentalisation and nuclear pore penetration specificity [61]. Heparin conjugated quantum dots were reported to have superior imaging properties compared to their native counterparts, facilitating QDs internalization into the nucleus of phagocytic, colonic and lung cancer cell lines [62]. Nuclear and nucleolar localization phenomenon characteristic of the negatively charged QDs (Fig. 2) could be possibly further exploited for selective manipulation of the cell cycle at the histone level [63].

More recently, Song et al. have shown that new emerging types of QDs, such as water-soluble graphene quantum dots can be applied as multiphoton fluorophore probes in live mouse primary hepatocytes [64]. Of note, no significant cytotoxicity was registered with these QDs concentrations of up to $100 \mu \mathrm{g} / \mathrm{ml}$. Yuan et al. [65] reported that graphene quantum dots offer remarkable advantages in biological applications as multicolour fluorescent probes sensitive to an entire $\mathrm{pH}$ range from 1 to 14 under naked eye detection. These QDs were also found to be responsive to the temperature changes, thereby rendering them useful dual probes for simultaneous $\mathrm{pH}$ and temperature measurements even in complex biological media. Graphene QDs used as energy donor with rhodamine derivative as an energy acceptor provide a ratiometric FRET sensor for estimation of the heavy metal $(\mathrm{Hg})$ levels in living cultured cells and such combination sensor features high selectivity, low cytotoxicity, biocompatibility and good water solubility [66]. Prabhakar

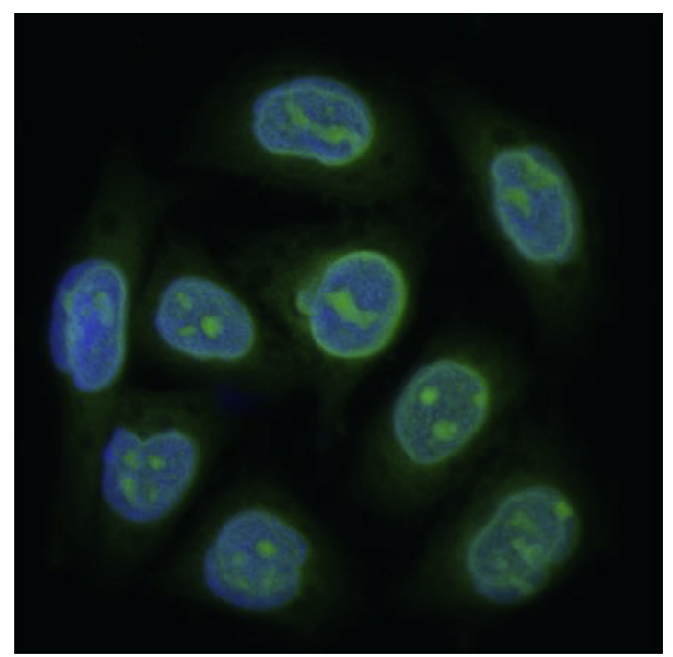

Fig. 2. Green-emitting negatively charged QDs highlighting nucleoli as the predominant localisation sites of intranuclear histones. 
et al. recently demonstrated that functionalized graphene oxide QDs with improved photoluminescence qualities are the promising candidates as optical probes for preclinical imaging [67]. The authors implemented functionalization of graphene oxide QDs with poly-ethyleneglycol/poly-ethylene imine and folic acid tag as the affinity ligand for tumour cells expressing folate receptors. These QDs featured low toxicity, were retained within the labelled cells population over eight days and were redistributed into dividing daughter cells. In the chicken embryo chorioallantoic membrane model the authors were also able to visualize and track migratory cancer cells, suggesting that these QDs could possibly overcome the limitations pertaining to "classical" semiconductor QDs in longterm biomedical imaging and in vivo application potential. Molybdenum sulphate QDs demonstrated good performance as probes for multiphoton imaging and have been successfully exploited to design the intracellular microRNA imaging analysis system [41].

Similar to semiconductor QDs, silicon-based QDs are highly fluorescent and in principle can be used for cellular imaging, but in native form are either not soluble in water or their fluorescence rapidly deteriorates in aqueous environment even after surface functionalization. Fan et al. constructed blue- and green-emitting Si QDs which proved to be photostable, biocompatible and underwent a rapid distribution throughout cytoplasm, with a significant fraction also entering the nucleus of cultured monocytes [68]. Silicon $\left(\mathrm{Si} / \mathrm{SiO}_{2}\right)$ quantum dots were recently used to study nanoparticle uptake and inflammation process in human lung fibroblastic line MRC-5 [69].

QDs-decorated gold nanorods with silicon coating functionalized with transferrin have been taken up by A549 lung cancer cell line and demonstrated good potential as high contrast fluorescent/ plasmonic bimodal labels for fluorescence and dark-field cell imaging [70]. A highly sensitive electro-chemiluminescent assay based on double-quenching of graphene QDs by G-quadruplexhemin and gold nanoparticles for protein kinase A activity detection in serum samples and cell lysates with a detection limit down to $0.04 \mathrm{U} / \mathrm{mL}$ was designed by Liu et al. [71], which might be potentially utilised both in experimental settings and clinical biochemistry diagnostics.

\section{In vivo imaging using quantum dots}

Application of QDs as contrast agents for in vivo imaging has been the area of high expectations since the initial studies carried out over a decade ago and has recently received a recurring attention. Organic fluorophores, fluorescent and chemiluminescent probes are still the most commonly used optical reporter molecules for animal imaging at present [72-74]. However, conventional organic probes do not offer the near-infrared (>650 nm) emission possibility. The near-infrared emitting window is very appealing for biomedical imaging because of the low tissue absorption and reduced light scattering in this wavelengths' region [75,76]. Since the optical properties of QDs can be tuned by size and composition, $\mathrm{CdTe}, \mathrm{CdTeSe}$, InPAs, $\mathrm{PbS}$, and PbSe featuring near-infrared emission have been produced and applied for bio-imaging purposes [73,77-79]. Several earlier publications demonstrated QDs as in vivo contrast agents possessing important advanced properties. Alloyed semiconductor QDs have been successfully synthesized to achieve red-shifted light emission up to $850 \mathrm{~nm}$ and quantum yields up to $60 \%$ without changing the particle size [77]. QDs were tuned to NIR fluorescence emission (emitting at $850 \mathrm{~nm}$ ) and utilized in major cancer surgery for mapping sentinel lymph nodes at the depth down to $1 \mathrm{~cm}$ [78-80]. Protease-activated NIR fluorescent probes were developed to detect tumour-associated lysosomal protease activity associated with the development of early stage tumours in vivo in a mouse xenograft model [81]. In earlier studies,
Akerman et al. functionalized ZnS-capped CdSe QDs with peptides enabling targeting of tumours, blood and lymphatic vessels in tumour-bearing mice [82]. QDs-labelled cancer cells and fluorescence emission-scanning microscopy were used to track the extravasation and spreading of malignant cells into lung tissue in mice following intravenous administration [83]. So et al. presented QDs conjugates that luminesce by resonance energy transfer in the absence of external excitation and confirmed that the conjugates emitted long-wavelength (from red to near-infrared) bioluminescent light in cells and were suitable for multiplexed in vivo imaging even in deep animal tissue locations [84]. Han et al. have recently developed novel fluorophores based on QDs-antibodies conjugates coated with norbornene-displaying polyimidazole ligands. These multiplexed probes were applied for intravital cytometric in vivo imaging and single-cell labelling in bone marrow. Using a calvarial bone window system, the authors showed that the QD conjugates efficiently diffused into the bone marrow and labelled single cells of rare populations of hematopoietic stem and progenitor cells [85]. Shi et al. have designed the multifunctional graphene oxide QDsdecorated magnetic nanoplatform enabling the challenging task of capturing rare Glypican-3-expressing Hep G2 circulating liver cancer tumour cells in tumour cell-enriched blood preparations containing as few as 10 cancer cells in $15 \mathrm{~mL}$ [86]. Their two-photon luminescence platform was suitable for subsequent selective cancer cells imaging in a biological transparency window using the $960 \mathrm{~nm}$ wavelength light. Recent in vitro and in vivo toxicity studies demonstrated the unique biocompatibility of optimised Si QDs and fluorescent nanodiamonds, positioning them as excellent candidates for diagnostic imaging and promising non-toxic vectors for therapeutic drug delivery [22]. Composite silica-coated gold nanosphere/quantum dots nanoparticles (Au-SiO2-QDs/SiO2-PVP) produced by Song et al. [87] were implemented as dual mode probes for contrast-enhanced X-ray CT and fluorescence imaging.

\section{Quantum dots for diagnostics and therapy}

The development of efficient applications of QDs for diagnostic and therapeutic purposes is heavily dependent on the availability of nanoparticles small enough to cross the multiple biological barriers imposed by the tissues and organs of the human body, such as blood-brain, alveolo-capillary, intestinal and dermal barriers and to be excreted with urine through the renal filtration barrier, i.e. they must be below $8-10 \mathrm{~nm}$ at least in the core diameter (usually significantly increased by the addition of surface functionalities and adsorption of plasma proteins). In addition, they must demonstrate low nonspecific cytotoxicity levels. On the other hand, multifunctional QDs, as all other rigid nanoparticle-based drug carriers offer a potential to exploit the enhanced permeability and retention, or EPR, effect characterised by the more "leaky" vasculature found in tumour compared to the normal tissue [88] and, in principle to expect similar increased accumulation of QDs-conjugated therapeutic drugs over the targeted cancer lesion. Unfortunately, the multifunctional nanosystems designed for theranostic purposes and possessing the most exciting potential in nanomedicine in realistic application scenarios frequently fall below the original expectations either due to the unsatisfactory targeting properties, poor payload and release of the conjugated therapeutic drugs, or both. From this prospective, the ex vivo QDs-based diagnostic systems and devices currently have reached a far more advanced stage than the nanosystems for whole body imaging and/or targeted drug delivery.

QDs are perfectly suitable for protein microarrays technology representing an invaluable tool for detection of proteins, protein-protein interactions and responses to therapeutic drugs [89]. Such systems are especially useful for early diagnosis of cancer, as it 
can provide a better prognosis and timely implementation of appropriate treatment schemes for the patients. The major drawback of traditional biomarker screening techniques is that they commonly detect a single molecular target per assay. A fast and low cost QDs-based lab-on-a-bead immunodiagnostic multiplexed system has been recently offered and pre-clinically validated for the detection of multiple prostate-specific cancer biomarkers in clinical serum samples [90]. Notably, the sensitivity and specificity of the system proved to be similar to the gold-standard ELISA assays.

QDs conjugated with mutation-specific antibodies were recently used for immunofluorescence histochemical detection of epidermal growth factor (EGFR) gene mutations in the non-small cell lung cancer clinical samples by Qu et al., who demonstrated that QDs-immunohistochemical approach provides a simple and standardized method to detect EGFR mutations with high sensitivity and specificity, as compared with real-time polymerase chain reaction. From this perspective, the development of specific antibodies against the mutated EGFR proteins might in future applied for the diagnostics and treatment of lung cancer [91].

Johari-Aharet et al. have designed an ultra-sensitive impedimetric immunosensor for detection of the serum biomarker CA-125 in patients with ovarian cancer. For this purpose, they modified a gold electrode with mercaptopropionic acid and subsequently conjugated it with silica coated gold nanoparticles, CdSe QDs and anti-CA-125 monoclonal antibody. The authors report a sensitive detection of CA-125 with a detection limit as low as $0.0016 \mathrm{U} / \mathrm{mL}$ featuring linearity over $0-0.1 \mathrm{U} / \mathrm{mL}$ [92].

Sukhanova et al. demonstrated a successful application of nanocrystal-encoded microbeads to clinical proteomics in detection of circulating autoantibodies serving as markers of systemic sclerosis [93].

The field of cancer diagnostics deservedly keeps attracting the attention of researches, and this area has currently shown the outstanding potential for realistic clinical translation. QDs and nanocrystal-encoded microbeads coupled with antibodies against tumour markers can be useful tools for ultra-sensitive micrometastases detection in serum and tissues [94]. Single domain antibodies-quantum dot conjugates, being much smaller in size compared to similar conventional antibody-based systems, hence offering a deeper tissue penetration in clinical biopsy samples, have been shown to be promising candidates for detection of lung and breast cancer biomarkers [95].

For potential clinical coagulation and haemostasis laboratory use, a novel promising tool may be presented by bi-functionalized aptasensor for thrombin detection incorporating CdSe QDs and implementing electrochemiluminescence and electrochemical impedance spectroscopy fabricated by Lu et al. [96]. In thrombincontaining sample the aptamer combines with this essential coagulation-related protein, resulting in loss of QDs from the electrode surface, decreasing the electrochemiluminescence intensity and increasing the impedance. Such bifunctionalized aptasensor demonstrated high selectivity and super sensitivity with a detection limit of $10^{-10} \mu \mathrm{g} / \mathrm{mL}$ or $2.7 \mathrm{aM}$.

From the clinical microbiology diagnostic perspective, an interesting innovation is offered by the combination magnetic/silica nanotubes surface-modified with QDs for simultaneous separation and highly sensitive detection of pathogenic Escherichia coli and Salmonella enteritis typhimurium bacteria in the range of $10^{2}-10^{5} \mathrm{CFU} / \mathrm{ml}$ [97].

Graphene is considered to be an attractive candidate for nanomedical applications, including NIR-responsive cancer therapy [21]. A dual-mode surface enhanced Raman scattering and fluorescence immunoassay using graphene QDs labels on one-dimensional aligned magneto-plasmonic nanoparticles has been recently developed by Zou et al. [98] and used for the diagnostic detection of tuberculosis antigen CFP-10, reaching the excellent analyte detection limit of $0.0511 \mathrm{pg} / \mathrm{mL}$. Dong et al. developed multifunctional poly(L-lactide)-polyethylene glycol-grafted photoluminescent graphene quantum dots for intracellular microRNA imaging in HeLa cells [99]. They suggested the use of such nanosystems in clinical practice for gene delivery with enhanced therapeutic efficiency, as they feature high biocompatibility and low cytotoxicity. Many other emerging applications of graphene-based nanomaterials for molecular imaging, including theranostics have been recently overviewed by Garg et al. [100].

Ohta et al. [101] utilised amine-modified silicon quantum dots with visible photoluminescence as building blocks, assembling drug-loaded Si QDs aggregates. The aggregates underwent a disruption in response to the low endosomal $\mathrm{pH}$, hence enabling the selective intracellular release of the loaded drugs [101].

The above mentioned "quantum rattles" based on gold QDs designed by Hembury et al. have been shown to be capable of reducing tumour burden in a single course of photothermal therapy in vivo while coupling three complementary imaging modalities, such as near-infrared fluorescence, photoacoustic and magnetic resonance imaging and facilitating the function of the silica shell as a drug carrier [43]. Enhanced photosensitizer properties of hybrid methylene blue and CdTe nanocrystals systems make them attractive candidates for photodynamic therapy [102]. From this perspective, gold and many other types of QDs can serve as versatile nanoscale scaffolds for designing multifunctional nanoparticles with simultaneous imaging and therapeutic functionalities [103].

The reports on the application of QDs for regenerative medicine as another nanomedicine domain are currently limited to a couple of dozens and are mostly related to testing of the innovative tissue engineering systems performance $[104,105]$ or stem cells tracking using QDs as fluorescent labels [106,107] and therefore are not discussed in detail in the current review.

\section{Unresolved problems, challenges and controversies}

One of the key problems facing the researchers aiming to develop QDs for nanomedical uses was and remains the frequently encountered and often high toxicity levels of these nanoparticles. But if earlier it was commonly attributed to the "classical" semiconductor nanocrystals composed of such inherently hazardous metals as $\mathrm{Cd}, \mathrm{Pb}$ and similar, the growing amount of data bears witness that this problem is, to a various extent, also pertinent to many new emerging types of QDs and the oxidative stress appears to be the common key factor involved in the cytotoxicity of these nanomaterials.

Thus, $\mathrm{Si} / \mathrm{SiO} 2$ QDs have been reported to cause cytotoxicity in lung cells [108]. In this study, Stan et al. demonstrated for the first time the negative influence of silicon QDs on cellular redox homeostasis and glutathione distribution in human MRC-5 lung fibroblastic cell line. This was reflected in the increased levels of reactive oxygen species and malondialdehyde along with the lowered glutathione content. Interestingly, they observed that the initial localisation of glutathione in the cytoplasm has been gradually shifting to the nucleus over longer incubation intervals, suggesting a complex multi-step mechanism of this process. The same group has also shown that silicon-based QDs are able to generate inflammation in lung cells and cause an imbalance in extracellular matrix turnover through a differential regulation of matrix metalloproteinases and tissue inhibitor of metalloproteinase- 1 protein expression [69].

Qin et al. investigated the effects of graphene QDs on macrophages and their underlying molecular mechanisms. Their study 
has shown that graphene QDs only marginally affected the cell viability and cell membrane integrity of macrophages, while significantly increasing reactive oxygen species generation, apoptotic cell death with associated raised expression levels of Bax, Bad, caspase 3, caspase 9, beclin 1, and LC3-I/II, the latter indicating autophagy as an additional recently documented nanotoxicityrelated mechanism. Low concentrations of graphene QDs also significantly enhanced the expression of several cytokines, such as tumour necrosis factor- $\alpha$, interleukin- $1 \beta$ and IL- 8 in a p38 mitogenactivated protein kinase MAPK-dependent manner, whereas high concentrations of these QDs elicited opposite effects on the cytokines production [109].

Graphene QDs proved to be toxic also in in vivo zebrafish embryo model at concentrations exceeding $25 \mu \mathrm{g} / \mathrm{mL}$ [110]. Cytotoxicity and genotoxicity of graphene QDs was studied by Wang et al. in fibroblastic cell line NIH-3T3 cells. Cells exposed to graphene QDs at concentrations over $50 \mu \mathrm{g} / \mathrm{mL}(-1)$ showed no significant cytotoxicity, but the increased expression of p53, Rad 51, and OGG1 proteins reflecting the DNA damage by reactive oxygen species was recorded [111].

Despite a crucial importance of understanding the mechanisms of nanoparticle-associated side effects on the blood components which are likely to develop following potential systemic administration of nano-enabled imaging probes and drug nanocarriers (Fig. 3), so far only a limited number of experimental studies has been carried out demonstrating the coagulation-modifying side effects of QDs [112-115].

Mice injected with amine and carboxyl modified CdSe/ZnS QDs rapidly developed pulmonary vascular thrombosis and a nearly instantaneous death [112]. Carboxyl-functionalised QDs were more potent than their amine counterparts in inducing pulmonary vascular thrombosis. This leads to the suggestion that the carboxyl QDs activate the coagulation cascade through the intrinsic pathway, since the thrombotic effect was not detected following pre-treatment of mice with heparin. These results correlate well with the reports that CdSe QDs can induce platelet aggregation in vitro $[115,116]$. The dramatic effects of different nanoparticle types, including QDs, on the coagulation cascade are bound to develop as a hot topic in the focus of attention of research groups worldwide.

Since the initial discovery and introduction of the term "protein corona" as a dynamic protein assembly on the surface of nanoparticles exposed to complex biological media [117-119], numerous studies have confirmed its importance for the

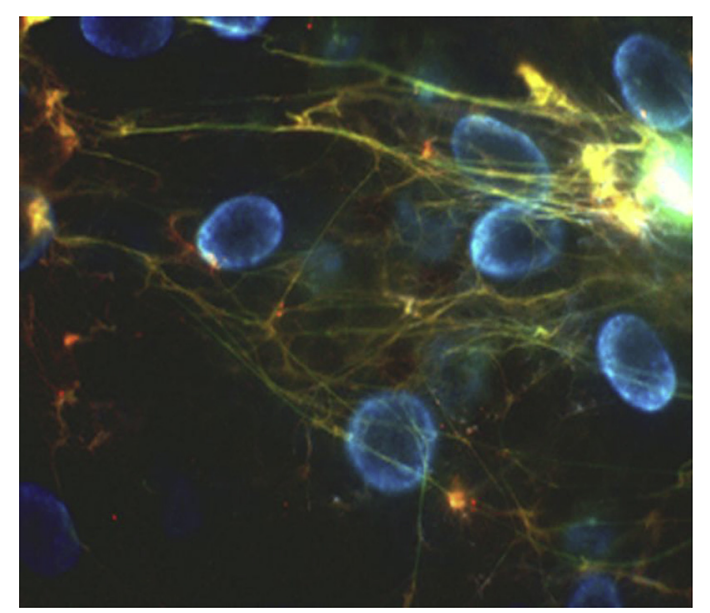

Fig. 3. Negatively charged unmodified CdTe QDs decorating fibrin (green/yellow fibres) in the fresh human blood clot. modification of functional properties of nanoparticles, their cellular, tissue and organ distribution patterns and for the overall outcome of cell interactions with them. Surprisingly, QDs received a far lesser attention in this regard. A possible explanation of this is possibly associated with the fact protein corona formation is often addressed to and generalised out of context of QDs and other nanoparticles of similar extremely small sizes (below $5 \mathrm{~nm}$ ), comparable to or even smaller that the size of most common proteins. It is reasonable to suggest that approaching such dimensions, the tiniest QDs are highly unlikely to be decorated with a proper protein "corona", but would rather themselves form a kind of intercalating "nano-glitter" on the protein surface and/or within the folded protein structures at the sites which are favourable for the QDs-protein interactions both in terms of the physical mechanisms such as charge, hydrophobicity level, the nature of available chemical groups and bonds for such interactions and size constrains [120]. Some of these interactions where the macromolecular scaffolds can serve as templates for ultra-small nanoparticles deposition and resulting changes in protein folding (summarised by Shemetov et al. [121]), are bound to have profound bilateral effects both of protein and QDs properties. Indeed, Pathak et al. more recently studied the interactions between water-soluble MPA-coated semiconductor CdSe QDs and three globular plasma proteins, including bovine serum albumin, $\beta$-lactoglobulin and human serum albumin and found that acidic residues of these proteins engaged in electrostatic interactions with QDs and upon binding with QDs, protein molecules underwent substantial conformational changes at the secondary-structure level with up to $50 \%$ loss in helicity [122]. Maffre et al. investigated the adsorption of human serum albumin onto Fe-Pt nanoparticles, CdSe/ZnS QDs, Au and Ag nanoclusters of 6,5 and 1-4 nm radii, respectively. The authors implemented different water-solubilizing approaches exposing carboxyl, amino groups and their combinations as well as succinylation and amination to render nanoparticles with different surface charges. The affinity of human serum albumin towards these nanoparticles differed by almost four orders of magnitude [123]. Treuel et al. investigated the protein corona formation around larger $10 \mathrm{~nm}$ diameter dihydrolipoic acid-coated QDs. Modification of human serum albumin molecules by introducing additional carboxyl groups resulted in a 3-fold decreased binding affinity towards the QDs. In contrast, a 1000-fold enhanced affinity was observed for albumin modified by ethylenediamine increasing the number of amino functions on the protein surface. In this study, pronounced variations were also observed both in the protein corona physicochemical properties and subsequent QDs uptake by HeLa cells [124].

Therefore, it is clear that further studies are required to gain an in-depth understanding of the specifics of interactions between biological macromolecules and QDs, facilitating their rational design as ideal tools for nanomedical applications. In addition, a safe-by-design multi-tier approach with systematic tests conducted at each stage of QDs preparation and modification for biomedical use recently suggested by Movia et al. [125] should be adhered to in order to provide a realistic chance for their future clinical translation.

\section{Conclusions and perspectives}

The analysis of the results of the recent studies focused on applications of QDs in nanomedicine demonstrates that these nanoparticles have an outstanding potential for medical research, diagnostics and innovative methods of drug delivery. The already available prototype diagnostic tools feature a high feasibility for clinical translation in the near future. Despite the arrival of new emerging nanoparticles into the class of QDs featuring advanced 
properties and reduced cytotoxicity, detailed physico-chemical and functional characterisation of the QDs-based systems require further in-depth investigations to ensure their expected efficiency and safety in in vivo application scenarios in humans. From the investment prospective, according to the recent Allied Market Research Report on Quantum Dots (Global Analysis, Growth, Trends, Opportunities, Size, Share and Forecast through 2020), the global QDs market is expected to grow from \$316 million in 2013 to $\$ 5040$ million in 2020 with a projected growth rate of nearly 30\% per annum. The QDs market segment related to biological imaging is the most mature in terms of revenue and it is expected to undergo consistent growth. The end-user segment representing QDsbased medical devices currently has the highest market share as medical scientists have been engaged in developing these devices for more than two decades by now [126]. Taken together, these research achievements and economic trends provide an overall strong and optimistic foundation for the future of QDs on the nanomedicine landscape.

\section{Acknowledgements}

This work was partially supported by the EU FP7 NAMDIATREAM project (NMP-2009-LARGE-3-246479) and MULTIFUN project (NMP-2010-LARGE-4-262943).

\section{Transparency document}

Transparency document related to this article can be found online at http://dx.doi.org/10.1016/j.bbrc.2015.07.039.

\section{References}

[1] A. Hoshino, S. Hanada, K. Yamamoto, Toxicity of nanocrystal quantum dots: the relevance of surface modifications, Arch. Toxicol. 85 (2011) 707-720.

[2] A. Tan, L. Yildirimer, J. Rajadas, H. De La Pena, G. Pastorin, A. Seifalian, Quantum dots and carbon nanotubes in oncology: a review on emerging theranostic applications in nanomedicine, Nanomedicine (Lond) 6 (2011) $1101-1114$

[3] C. Chen, J. Peng, S.R. Sun, C.W. Peng, Y. Li, D.W. Pang, Tapping the potential of quantum dots for personalized oncology: current status and future perspectives, Nanomedicine (Lond) 7 (2012) 411-428.

[4] C.L. Grigsby, Y.P. Ho, K.W. Leong, Understanding nonviral nucleic acid delivery with quantum dot-FRET nanosensors, Nanomedicine (Lond) 7 (2012) $565-577$.

[5] G. Bao, S. Mitragotri, S. Tong, Multifunctional nanoparticles for drug delivery and molecular imaging, Annu. Rev. Biomed. Eng. 15 (2013) 253-282.

[6] S.Y. Madani, F. Shabani, M.V. Dwek, A.M. Seifalian, Conjugation of quantum dots on carbon nanotubes for medical diagnosis and treatment, Int. J. Nanomed. 8 (2013) 941-950.

[7] J. Conde, J.T. Dias, V. Grazu, M. Moros, P.V. Baptista, J.M. de la Fuente, Revisiting 30 years of biofunctionalization and surface chemistry of inorganic nanoparticles for nanomedicine, Front. Chem. 2 (2014) 48.

[8] C.S. Henderson, A.C. Madison, A. Shah, Size matters-nanotechnology and therapeutics in rheumatology and immunology, Curr. Rheumatol. Rev. 10 (2014) $11-21$.

[9] X. Yi, F. Wang, W. Qin, X. Yang, J. Yuan, Near-infrared fluorescent probes in cancer imaging and therapy: an emerging field, Int. J. Nanomed. 9 (2014) $1347-1365$.

[10] O.S. Wolfbeis, An overview of nanoparticles commonly used in fluorescent bioimaging, Chem. Soc. Rev. 44 (2015) 4743-4768.

[11] X. Michalet, F.F. Pinaud, L.A. Bentolila, J.M. Tsay, S. Doose, J.J. Li, G. Sundaresan, A.M. Wu, S.S. Gambhir, S. Weiss, Quantum dots for live cells, in vivo imaging, and diagnostics, Science 307 (2005) 538-544.

[12] M. Ferrari, Cancer nanotechnology: opportunities and challenges, Nat. Rev. Cancer 5 (2005) 161-171.

[13] I.L. Medintz, A.R. Clapp, H. Mattoussi, E.R. Goldman, B. Fisher, J.M. Mauro, Self-assembled nanoscale biosensors based on quantum dot FRET donors, Nat. Mater. 2 (2003) 630-638.

[14] E.R. Goldman, A.R. Clapp, G.P. Anderson, H.T. Uyeda, J.M. Mauro, I.L. Medintz, $\mathrm{H}$. Mattoussi, Multiplexed toxin analysis using four colors of quantum dot fluororeagents, Anal. Chem. 76 (2004) 684-688.

[15] W.B. Cai, D.W. Shin, K. Chen, O. Gheysens, Q.Z. Cao, S.X. Wang, S.S. Gambhir X.Y. Chen, Peptide-labeled near-infrared quantum dots for imaging tumor vasculature in living subjects, Nano Lett. 6 (2006) 669-676.
[16] X.H. Gao, L.L. Yang, J.A. Petros, F.F. Marshal, J.W. Simons, S.M. Nie, In vivo molecular and cellular imaging with quantum dots, Curr. Opin. Biotechnol. 16 (2005) 63-72.

[17] S.M. Moghimi, A.C. Hunter, J.C. Murray, Nanomedicine: current status and future prospects, FASEB J. 19 (2005) 311-330.

[18] R. Bilan, F. Fleury, I. Nabiev, A. Sukhanova, Quantum dot surface chemistry and functionalization for cell targeting and imaging, Bioconjug. Chem. 26 (2015) 609-624.

[19] T.Q. Vu, W.Y. Lam, E.W. Hatch, D.S. Lidke, Quantum dots for quantitative imaging: from single molecules to tissue, Cell. Tissue Res. 360 (2015) 71-86.

[20] J. Wen, Y. Xu, H. Li, A. Lu, S. Sun, Recent applications of carbon nanomaterials in fluorescence biosensing and bioimaging, Chem. Commun. (Camb) 51 (2015) 11346-11358.

[21] J.M. Yoo, J.H. Kang, B.H. Hong, Graphene-based nanomaterials for versatile imaging studies, Chem. Soc. Rev. 44 (2015) 4835-4852.

[22] M. Montalti, A. Cantelli, G. Battistelli, Nanodiamonds and silicon quantum dots: ultrastable and biocompatible luminescent nanoprobes for long-term bioimaging, Chem. Soc. Rev. 44 (2015) 4853-4921.

[23] M.A. Reed, J.N. Randall, R.J. Aggarwal, R.J. Matyi, T.M. Moore, A.E. Wetsel, Observation of discrete electronic states in a zero-dimensional semiconductor nanostructure, Phys. Rev. Lett. 60 (1988) 535-537.

[24] P. Alivisatos, The use of nanocrystals in biological detection, Nat. Biotechnol. 22 (2004) 47-52.

[25] L.A. Bentolila, Y. Ebenstein, S. Weiss, Quantum dots for in vivo small-animal imaging, J. Nucl. Med. 50 (2009) 493-496.

[26] H.M. Azzazy, M.M. Mansour, S.C. Kazmierczak, From diagnostics to therapy: prospects of quantum dots, Clin. Biochem. 40 (2007) 917-927.

[27] E.R. Goldman, I.L. Medintz, H. Mattoussi, Luminescent quantum dots in immunoassays, Anal. Bioanal. Chem. 384 (2006) 560-563.

[28] K.K. Jain, Applications of nanobiotechnology in clinical diagnostics, Clin. Chem. 53 (2007) 2002-2009.

[29] A.P. Alivisatos, W. Gu, C. Larabell, Quantum dots as cellular probes, Annu Rev. Biomed. Eng. 7 (2005) 55-76,

[30] A.M. Smith, G. Ruan, M.N. Rhyner. S. Nie, Engineering luminescent quantum dots for in vivo molecular and cellular imaging, Ann. Biomed. Eng. 34 (2006) 3-14.

[31] S. Dey, Y. Zhou, X. Tian, J.A. Jenkins, O. Chen, S. Zou, J. Zhao, An experimental and theoretical mechanistic study of biexciton quantum yield enhancement in single quantum dots near gold nanoparticles, Nanoscale 7 (2015) 6851-6858.

[32] Y. Yin, A.P. Alivisatos, Colloidal nanocrystal synthesis and the organicinorganic interface, Nature 437 (2005) 664-670.

[33] D. Maysinger, J. Lovric, A. Eisenberg, R. Savic, Fate of micelles and quantum dots in cells, Eur. J. Pharm. Biopharm. 65 (2007) 270-281.

[34] A.R. Clapp, E.R. Goldman, H. Mattoussi, Capping of CdSe-ZnS quantum dots with DHLA and subsequent conjugation with proteins, Nat. Protoc. 1 (2006) $1258-1266$.

[35] X. Gao, Y. Cui, R.M. Levenson, L.W. Chung, S. Nie, In vivo cancer targeting and imaging with semiconductor quantum dots, Nat. Biotechnol. 22 (2004) 969-976.

[36] X. Wu, H. Liu, J. Liu, K.N. Haley, J.A. Treadway, J.P. Larson, N. Ge, F. Peale, M.P. Bruchez, Immunofluorescent labeling of cancer marker HER2 and other cellular targets with semiconductor quantum dots, Nat. Biotechnol. 21 (2003) 41-46.

[37] B. Dubertret, P. Skourides, D.J. Norris, V. Noireaux, A.H. Brivanlou, A. Libchaber, In vivo imaging of quantum dots encapsulated in phospholipid micelles, Science 298 (2002) 1759-1762.

[38] N. Gaponik, D.V. Talapin, A.L. Rogach, K. Hoppe, E.V. Shevchenko, A. Kornowski, A. Eychmüller, H. Weller, Thiol-capping of CdTe nanocrystals: an alternative to organometallic synthetic routes, J. Phys. Chem. B 106 (2002) 7177-7185.

[39] S. Kundu, R.M. Yadav, T.N. Narayanan, M.V. Shelke, R. Vajtai, P.M. Ajayan, V.K. Pillai, Synthesis of N, F and S co-doped graphene quantum dots, Nanoscale (2015), http://dx.doi.org/10.1039/C5NR02427G.

[40] C.K. Chua, Z. Sofer, P. Simek, O. Jankovsky, K. Klimova, S. Bakardjieva, S. Hrdlickova Kuckova, M. Pumera, Synthesis of strongly fluorescent graphene quantum dots by cage-opening buckminsterfullerene, ACS Nano 9 (2015) 2548-2555.

[41] W. Dai, H. Dong, B. Fugetsu, Y. Cao, H. Lu, X. Ma, X. Zhang, Tunable fabrication of molybdenum disulfide quantum dots for intracellular microRNA detection and multiphoton bioimaging, Small (2015), http://dx.doi.org/10.1002/ smll.201500208.

[42] Y. Zhong, X. Sun, S. Wang, F. Peng, F. Bao, Y. Su, Y. Li, S.T. Lee, Y. He, Facile, large-quantity synthesis of stable, tunable-color silicon nanoparticles and their application for long-term cellular imaging, ACS Nano 9 (2015) $5958-5967$.

[43] M. Hembury, C. Chiappini, S. Bertazzo, T. L Kalber, G. L Drisko, O. Ogunlade, S. Walker-Samuel, K.S. Krishna, C. Jumeaux, P. Beard, C.S. Kumar, A.E. Porter, M.F. Lythgoe, C. Boissiere, C. Sanchez, M.M. Stevens, Gold-silica quantum rattles for multimodal imaging and therapy, Proc. Natl. Acad. Sci. U. S. A. 112 (2015) 1959-1964.

[44] J. Zheng, C.W. Zhang, R.M. Dickson, Highly fluorescent, water-soluble, sizetunable gold quantum dots, Phys. Rev. Lett. 93 (2004).

[45] M. Geszke-Moritz, M. Moritz, Quantum dots as versatile probes in medical sciences: synthesis, modification and properties, Mater. Sci. Eng. C Mater. Biol. Appl. 33 (2013) 1008-1021. 
[46] I.L. Medintz, H.T. Uyeda, E.R. Goldman, H. Mattoussi, Quantum dot bioconjugates for imaging, labelling and sensing, Nat. Mater. 4 (2005) 435-446.

[47] E.R. Goldman, E.D. Balighian, H. Mattoussi, M.K. Kuno, J.M. Mauro, P.T. Tran, G.P. Anderson, Avidin: a natural bridge for quantum dot-antibody conjugates, J. Am. Chem. Soc. 124 (2002) 6378-6382.

[48] S.J. Rosenthal, I. Tomlinson, E.M. Adkins, S. Schroeter, S. Adams, L. Swafford, J. McBride, Y. Wang, L.J. DeFelice, R.D. Blakely, Targeting cell surface receptors with ligand-conjugated nanocrystals, J. Am. Chem. Soc. 124 (2002) 4586-4594.

[49] M. Xie, H.H. Liu, P. Chen, Z.L. Zhang, X.H. Wang, Z.X. Xie, Y.M. Du, B.O. Pan, D.W. Pang, CdSe/ZnS-labeled carboxymethyl chitosan as a bioprobe for live cell imaging, Chem. Commun. (Camb) (2005) 5518-5520.

[50] F. Pinaud, D. King, H.P. Moore, S. Weiss, Bioactivation and cell targeting of semiconductor $\mathrm{CdSe} / \mathrm{ZnS}$ nanocrystals with phytochelatin-related peptides, J. Am. Chem. Soc. 126 (2004) 6115-6123.

[51] P. De Koninck, S. Labrecque, C.D. Heyes, P.W. Wiseman, Probing synaptic signaling with quantum dots, HFSP J. 1 (2007) 5-10.

[52] X. Gao, S. Nie, Quantum dot-encoded mesoporous beads with high brightness and uniformity: rapid readout using flow cytometry, Anal. Chem. 76 (2004) 2406-2410.

[53] S. Pathak, S.K. Choi, N. Arnheim, M.E. Thompson, Hydroxylated quantum dots as luminescent probes for in situ hybridization, J. Am. Chem. Soc. 123 (2001) 4103-4104.

[54] H.M. Azzazy, M.M. Mansour, In vitro diagnostic prospects of nanoparticles, Clin. Chim. Acta 403 (2009) 1-8.

[55] L. Zhu, S. Ang, W.T. Liu, Quantum dots as a novel immunofluorescent detection system for Cryptosporidium parvum and Giardia lamblia, Appl. Environ. Microbiol. 70 (2004) 597-598.

[56] R. Edgar, M. McKinstry, J. Hwang, A.B. Oppenheim, R.A. Fekete, G. Giulian, C. Merril, K. Nagashima, S. Adhya, High-sensitivity bacterial detection using biotin-tagged phage and quantum-dot nanocomplexes, Proc. Natl. Acad. Sci. U. S. A. 103 (2006) 4841-4845.

[57] M. Han, X. Gao, J.Z. Su, S. Nie, Quantum-dot-tagged microbeads for multiplexed optical coding of biomolecules, Nat. Biotechnol. 19 (2001) 631-635.

[58] X. Gao, W.C. Chan, S. Nie, Quantum-dot nanocrystals for ultrasensitive biological labeling and multicolor optical encoding, J. Biomed. Opt. 7 (2002) $532-537$.

[59] A. Shamirian, A. Ghai, P.T. Snee, QD-Based FRET probes at a glance, Sensors (Basel) 15 (2015) 13028-13051.

[60] D. Maysinger, E. Hutter, Nanoparticle-based caspase sensors, Nanomedicine (Lond) 10 (2015) 483-501.

[61] Y. Williams, A. Sukhanova, M. Nowostawska, A.M. Davies, S. Mitchell, V. Oleinikov, Y. Gun'ko, I. Nabiev, D. Kelleher, Y. Volkov, Probing cell-typespecific intracellular nanoscale barriers using size-tuned quantum dots, Small 5 (2009) 2581-2588.

[62] C.M. Maguire, O.K. Mahfoud, T. Rakovich, V.A. Gerard, A. Prina-Mello, Y. Gun'ko, Y. Volkov, Heparin conjugated quantum dots for in vitro imaging applications, Nanomedicine 10 (2014) 1853-1861.

[63] J. Conroy, S.J. Byrne, Y.K. Gun'ko, Y.P. Rakovich, J.F. Donegan, A. Davies, D. Kelleher, Y. Volkov, CdTe nanoparticles display tropism to core histones and histone-rich cell organelles, Small 4 (2008) 2006-2015.

[64] S.H. Song, M.H. Jang, J.M. Jeong, H. Yoon, Y.H. Cho, W.I. Jeong, B.H. Kim, S. Jeon, Primary hepatocyte imaging by multiphoton luminescent graphene quantum dots, Chem. Commun. (Camb) 51 (2015) 8041-8043.

[65] F. Yuan, L. Ding, Y. Li, X. Li, L. Fan, S. Zhou, D. Fang, S. Yang, Multicolor fluorescent graphene quantum dots colorimetrically responsive to all-pH and a wide temperature range, Nanoscale 7 (2015) 11727-11733.

[66] M. Liu, T. Liu, Y. Li, H. Xu, B. Zheng, D. Wang, J. Du, D. Xiao, A FRET chemsensor based on graphene quantum dots for detecting and intracellular imaging of $\mathrm{Hg}(2$.$) , Talanta 143$ (2015) 442-449.

[67] N. Prabhakar, T. Nareoja, E. von Haartman, D. Sen Karaman, S.A. Burikov, T.A. Dolenko, T. Deguchi, V. Mamaeva, P.E. Hanninen, I.I. Vlasov, O.A. Shenderova, J.M. Rosenholm, Functionalization of graphene oxide nanostructures improves photoluminescence and facilitates their use as optical probes in preclinical imaging, Nanoscale 7 (2015) 10410-10420.

[68] J.W. Fan, R. Vankayala, C.L. Chang, C.H. Chang, C.S. Chiang, K.C. Hwang Preparation, cytotoxicity and in vivo bioimaging of highly luminescent water-soluble silicon quantum dots, Nanotechnology 26 (2015) 215703.

[69] M.S. Stan, C. Sima, L.O. Cinteza, A. Dinischiotu, Silicon-based quantum dots induce inflammation in human lung cells and disrupt extracellular matrix homeostasis, FEBS J. (2015), http://dx.doi.org/10.1111/febs.13330.

[70] Q. Wu, L. Chen, L. Huang, J. Wang, J. Liu, C. Hu, H. Han, Quantum dots decorated gold nanorod as fluorescent-plasmonic dual-modal contrasts agent for cancer imaging, Biosens. Bioelectron. 74 (2015) 16-23.

[71] J. Liu, X. He, K. Wang, D. He, Y. Wang, Y. Mao, H. Shi, L. Wen, A highly sensitive electrochemiluminescence assay for protein kinase based on doublequenching of graphene quantum dots by G-quadruplex-hemin and gold nanoparticles, Biosens. Bioelectron. 70 (2015) 54-60.

[72] C.H. Contag, B.D. Ross, It's not just about anatomy: in vivo bioluminescence imaging as an eyepiece into biology, J. Magn. Reson. Imag. 16 (2002) $378-387$.

[73] J. Klostranec, C.W. Chan, Quantum dots in biological and biomedical research: recent progress and present challenges, Adv. Mater. 18 (2006) 1953.

[74] R. Weissleder, Scaling down imaging: molecular mapping of cancer in mice, Nat. Rev. Cancer 2 (2002) 11-18.
[75] W. Jiang, E. Papa, H. Fischer, S. Mardyani, W.C. Chan, Semiconductor quantum dots as contrast agents for whole animal imaging, Trends Biotechnol. 22 (2004) 607-609.

[76] R. Weissleder, A clearer vision for in vivo imaging, Nat. Biotechnol. 19 (2001) 316-317.

[77] R.E. Bailey, S. Nie, Alloyed semiconductor quantum dots: tuning the optical properties without changing the particle size, J. Am. Chem. Soc. 125 (2003) 7100-7106.

[78] S. Kim, Y.T. Lim, E.G. Soltesz, A.M. De Grand, J. Lee, A. Nakayama, J.A. Parker, T. Mihaljevic, R.G. Laurence, D.M. Dor, L.H. Cohn, M.G. Bawendi, J.V. Frangioni, Near-infrared fluorescent type II quantum dots for sentinel lymph node mapping, Nat. Biotechnol. 22 (2004) 93-97.

[79] S.W. Kim, J.P. Zimmer, S. Ohnishi, J.B. Tracy, J.V. Frangioni, M.G. Bawendi, Engineering $\operatorname{InAs}(\mathrm{x}) \mathrm{P}(1-\mathrm{x}) / \mathrm{InP} / \mathrm{ZnSe}$ III-V alloyed core/shell quantum dots for the near-infrared, J. Am. Chem. Soc. 127 (2005) 10526-10532.

[80] J.V. Frangioni, S.W. Kim, S. Ohnishi, S. Kim, M.G. Bawendi, Sentinel lymph node mapping with type-II quantum dots, Methods Mol. Biol. 374 (2007) $147-159$.

[81] R. Weissleder, C.H. Tung, U. Mahmood, A. Bogdanov Jr., In vivo imaging of tumors with protease-activated near-infrared fluorescent probes, Nat. Biotechnol. 17 (1999) 375-378.

[82] M.E. Akerman, W.C. Chan, P. Laakkonen, S.N. Bhatia, E. Ruoslahti, Nanocrystal targeting in vivo, Proc. Natl. Acad. Sci. U. S. A. 99 (2002) 12617-12621.

[83] E.B. Voura, J.K. Jaiswal, H. Mattoussi, S.M. Simon, Tracking metastatic tumor cell extravasation with quantum dot nanocrystals and fluorescence emission-scanning microscopy, Nat. Med. 10 (2004) 993-998.

[84] M.K. So, C. Xu, A.M. Loening, S.S. Gambhir, J. Rao, Self-illuminating quantum dot conjugates for in vivo imaging, Nat. Biotechnol. 24 (2006) 339-343.

[85] H.S. Han, E. Niemeyer, Y. Huang, W.S. Kamoun, J.D. Martin, J. Bhaumik, Y. Chen, S. Roberge, J. Cui, M.R. Martin, D. Fukumura, R.K. Jain, M.G. Bawendi, D.G. Duda, Quantum dot/antibody conjugates for in vivo cytometric imaging in mice, Proc. Natl. Acad. Sci. U. S. A. 112 (2015) 1350-1355.

[86] Y. Shi, A. Pramanik, C. Tchounwou, F. Pedraza, R.A. Crouch, S.R. Chavva, A. Vangara, S.S. Sinha, S. Jones, D. Sardar, C. Hawker, P.C. Ray, Multifunctional biocompatible graphene oxide quantum dots decorated magnetic nanoplatform for efficient capture and two-photon imaging of rare tumor cells, ACS Appl. Mater. Interface 7 (2015) 10935-10943.

[87] J.T. Song, X.Q. Yang, X.S. Zhang, D.M. Yan, M.H. Yao, M.Y. Qin, Y.D. Zhao, Composite silica coated gold nanosphere and quantum dots nanoparticles for X-ray CT and fluorescence bimodal imaging, Dalton Trans. 44 (2015) $11314-11320$.

[88] H. Maeda, Toward a full understanding of the EPR effect in primary and metastatic tumors as well as issues related to its heterogeneity, Adv. Drug Deliv. Rev. (2015), http://dx.doi.org/10.1016/j.addr.2015.01.002.

[89] S. Krizkova, Z. Heger, M. Zalewska, A. Moulick, V. Adam, R. Kizek, Nanotechnologies in protein microarrays, Nanomedicine (Lond) (2015) 1-13.

[90] K. Brazhnik, Z. Sokolova, M. Baryshnikova, R. Bilan, A. Efimov, I. Nabiev, A. Sukhanova, Quantum dot-based lab-on-a-bead system for multiplexed detection of free and total prostate-specific antigens in clinical human serum samples, Nanomedicine 11 (2015) 1065-1075.

[91] Y.G. Qu, Q. Zhang, Q. Pan, X.D. Zhao, Y.H. Huang, F.C. Chen, H.L. Chen, Quantum dots immunofluorescence histochemical detection of EGFR gene mutations in the non-small cell lung cancers using mutation-specific antibodies, Int. J. Nanomed. 9 (2014) 5771-5778.

[92] M. Johari-Ahar, M.R. Rashidi, J. Barar, M. Aghaie, D. Mohammadnejad, A. Ramazani, P. Karami, G. Coukos, Y. Omidi, An ultra-sensitive impedimetric immunosensor for detection of the serum oncomarker CA-125 in ovarian cancer patients, Nanoscale 7 (2015) 3768-3779.

[93] A. Sukhanova, A.S. Susha, A. Bek, S. Mayilo, A.L. Rogach, J. Feldmann, V. Oleinikov, B. Reveil, B. Donvito, J.H. Cohen, I. Nabiev, Nanocrystal-encoded fluorescent microbeads for proteomics: antibody profiling and diagnostics of autoimmune diseases, Nano Lett. 7 (2007) 2322-2327.

[94] W. Mahmoud, A. Sukhanova, V. Oleinikov, Y.P. Rakovich, J.F. Donegan, M. Pluot, J.H. Cohen, Y. Volkov, I. Nabiev, Emerging applications of fluorescent nanocrystals quantum dots for micrometastases detection, Proteomics 10 (2010) 700-716

[95] T.Y. Rakovich, O.K. Mahfoud, B.M. Mohamed, A. Prina-Mello, K. CrosbieStaunton, T. Van den Broeck, L. De Kimpe, A. Sukhanova, D. Baty, A. Rakovich, S.A. Maier, F. Alves, F. Nauwelaers, I. Nabiev, P. Chames, Y. Volkov, Highly sensitive single domain antibody-quantum dot conjugates for detection of HER2 biomarker in lung and breast cancer cells, ACS Nano 8 (2014) $5682-5695$

[96] L. Lu, J. Li, T. Kang, S. Cheng, Bi-functionalized aptasensor for ultrasensitive detection of thrombin, Talanta 138 (2015) 273-278.

[97] P.D. Nguyen, S.J. Son, J. Min, Silica nanotube surface modification for multiplex detection of pathogenic bacteria, J. Nanosci. Nanotechnol. 14 (2014) $5646-5649$

[98] F. Zou, H. Zhou, T.V. Tan, J. Kim, K. Koh, J. Lee, Dual-mode SERS-fluorescence immunoassay using graphene quantum dot labeling on one-dimensional aligned magnetoplasmonic nanoparticles, ACS Appl. Mater. Interface 7 (2015) 12168-12175.

[99] H. Dong, W. Dai, H. Ju, H. Lu, S. Wang, L. Xu, S.F. Zhou, Y. Zhang, X. Zhang, Multifunctional poly(l-lactide)-polyethylene glycol-grafted graphene quantum dots for intracellular microRNA imaging and combined specific-gene- 
targeting agents delivery for improved therapeutics, ACS Appl. Mater. Interface 7 (2015) 11015-11023.

[100] B. Garg, C.H. Sung, Y.C. Ling, Graphene-based nanomaterials as molecular imaging agents, Wiley Interdiscip. Rev. Nanomed. Nanobiotechnol. (2015), http://dx.doi.org/10.1002/wnan.1342.

[101] S. Ohta, K. Yamura, S. Inasawa, Y. Yamaguchi, Aggregates of silicon quantum dots as a drug carrier: selective intracellular drug release based on $\mathrm{pH}$ responsive aggregation/dispersion, Chem. Commun. (Camb) 51 (2015) 6422-6425.

[102] A. Rakovich, D. Savateeva, T. Rakovich, J.F. Donegan, Y.P. Rakovich, V. Kelly V. Lesnyak, A. Eychmuller, CdTe quantum dot/dye hybrid system as photosensitizer for photodynamic therapy, Nanoscale Res. Lett. 5 (2010) 753-760.

[103] A.M. Smith, H. Duan, A.M. Mohs, S. Nie, Bioconjugated quantum dots fo in vivo molecular and cellular imaging, Adv. Drug Deliv. Rev. 60 (2008) $1226-1240$.

[104] T.J. Duncan, K. Baba, Y. Oie, K. Nishida, A novel method using quantum dots for testing the barrier function of cultured epithelial cell sheets, Invest Ophthalmol. Vis. Sci. 56 (2015) 2215-2223.

[105] A. Cunningham, H. Faircloth, M. Jones, C. Johnson, T. Coleman, G. Wicks, G. Postma, P. Weinberger, A reporter assay for the next generation of biomaterials: porous-wall hollow glass microspheres, Laryngoscope 124 (2014) 1392-1397.

[106] M.A. Hossain, T. Chowdhury, A. Bagul, Imaging modalities for the in vivo surveillance of mesenchymal stromal cells, J. Tissue Eng. Regen. Med. (2014), http://dx.doi.org/10.1002/term.1907.

[107] A. de Mel, J.T. Oh, B. Ramesh, A.M. Seifalian, Biofunctionalized quantum dots for live monitoring of stem cells: applications in regenerative medicine, Regen. Med. 7 (2012) 335-347.

[108] M.S. Stan, I. Memet, C. Sima, T. Popescu, V.S. Teodorescu, A. Hermenean, A. Dinischiotu, $\mathrm{Si} / \mathrm{SiO} 2$ quantum dots cause cytotoxicity in lung cells through redox homeostasis imbalance, Chem. Biol. Interact. 220 (2014) 102-115.

[109] Y. Qin, Z.W. Zhou, S.T. Pan, Z.X. He, X. Zhang, J.X. Qiu, W. Duan, T. Yang, S.F. Zhou, Graphene quantum dots induce apoptosis, autophagy, and inflammatory response via p38 mitogen-activated protein kinase and nuclear factor-kappaB mediated signaling pathways in activated THP-1 macrophages, Toxicology 327 (2015) 62-76.

[110] Z.G. Wang, R. Zhou, D. Jiang, J.E. Song, O. Xu, J. Si, Y.P. Chen, X. Zhou, L. Gan, J.Z. Li, H. Zhang, B. Liu, Toxicity of graphene quantum dots in zebrafish embryo, Biomed. Environ. Sci. 28 (2015) 341-351.

[111] D. Wang, L. Zhu, J.F. Chen, L. Dai, Can graphene quantum dots cause DNA damage in cells? Nanoscale 7 (2015) 9894-9901.

[112] J. Geys, A. Nemmar, E. Verbeken, E. Smolders, M. Ratoi, M. Hoylaerts B. Nemery, P. Hoet, Acute toxicity and prothrombotic effects of quantum dots: impact of surface charge, Environ. Health Perspect. 116 (2008) 1607-1613.

[113] Y.F. Loginova, N.I. Kazachkina, V.V. Zherdeva, A.L. Rusanov, M.V. Shirmanova E.V. Zagaynova, E.A. Sergeeva, S.V. Dezhurov, M.S. Wakstein, A.P. Savitsky, Biodistribution of intact fluorescent $\mathrm{CdSe} / \mathrm{CdS} / \mathrm{ZnS}$ quantum dots coated by mercaptopropionic acid after intravenous injection into mice, J. Biophot. 5 (2012) 848-859.
[114] S.P. Samuel, N. Jain, F. O'Dowd, T. Paul, D. Kashanin, V.A. Gerard, Y.K. Gun'ko, A. Prina-Mello, Y. Volkov, Multifactorial determinants that govern nanoparticle uptake by human endothelial cells under flow, Int. J. Nanomed. 7 (2012) 2943-2956.

[115] Y. Ramot, M. Steiner, V. Morad, S. Leibovitch, N. Amouyal, M.F. Cesta, A. Nyska, Pulmonary thrombosis in the mouse following intravenous administration of quantum dot-labeled mesenchymal cells, Nanotoxicology 4 (2010) 98-105.

[116] R. Dunpall, A.A. Nejo, V.S.R. Pullabhotla, A.R. Opoku, N. Revaprasadu, A. Shonhai, An in vitro assessment of the interaction of cadmium selenide quantum dots with DNA, iron, and blood platelets, IUBMB Life 64 (2012) 995-1002.

[117] T. Cedervall, I. Lynch, S. Lindman, T. Berggard, E. Thulin, H. Nilsson, K.A. Dawson, S. Linse, Understanding the nanoparticle-protein corona using methods to quantify exchange rates and affinities of proteins for nanoparticles, Proc. Natl. Acad. Sci. U. S. A. 104 (2007) 2050-2055.

[118] I. Lynch, T. Cedervall, M. Lundqvist, C. Cabaleiro-Lago, S. Linse, K.A. Dawson, The nanoparticle-protein complex as a biological entity; a complex fluids and surface science challenge for the 21st century, Adv. Colloid Interface Sci. 134-135 (2007) 167-174.

[119] M. Lundqvist, J. Stigler, G. Elia, I. Lynch, T. Cedervall, K.A. Dawson, Nanoparticle size and surface properties determine the protein corona with possible implications for biological impacts, Proc. Natl. Acad. Sci. U. S. A. 105 (2008) 14265-14270.

[120] A.E. Nel, L. Madler, D. Velegol, T. Xia, E.M. Hoek, P. Somasundaran, F. Klaessig, V. Castranova, M. Thompson, Understanding biophysicochemical interactions at the nano-bio interface, Nat. Mater. 8 (2009) 543-557.

[121] A.A. Shemetov, I. Nabiev, A. Sukhanova, Molecular interaction of proteins and peptides with nanoparticles, ACS Nano 6 (2012) 4585-4602.

[122] J. Pathak, K. Rawat, S. Sanwlani, H.B. Bohidar, Interaction of globular plasma proteins with water-soluble CdSe quantum dots, Chemphyschem 16 (2015) 1777-1786.

[123] P. Maffre, S. Brandholt, K. Nienhaus, L. Shang, W.J. Parak, G.U. Nienhaus, Effects of surface functionalization on the adsorption of human serum albumin onto nanoparticles - a fluorescence correlation spectroscopy study, Beilstein J. Nanotechnol. 5 (2014) 2036-2047.

[124] L. Treuel, S. Brandholt, P. Maffre, S. Wiegele, L. Shang, G.U. Nienhaus, Impact of protein modification on the protein corona on nanoparticles and nanoparticle-cell interactions, ACS Nano 8 (2014) 503-513.

[125] D. Movia, V. Gerard, C.M. Maguire, N. Jain, A.P. Bell, V. Nicolosi, T. O'Neill, D. Scholz, Y. Gun'ko, Y. Volkov, A. Prina-Mello, A safe-by-design approach to the development of gold nanoboxes as carriers for internalization into cancer cells, Biomaterials 35 (2014) 2543-2557.

[126] Marketsandmarkets.com, Quantum Dot Market by Product (Display, Medica Devices, Batteries, Solar Cells, Sensors, and Others) [online], Material, Application (Healthcare, Consumer, Defense, and Industry), and Geography-Forecast up to 2020, 2015. Available from: http://www.marketsandmarkets.com/MarketReports/quantum-dots-qd-market-694.html (accessed 30.06.2015). 